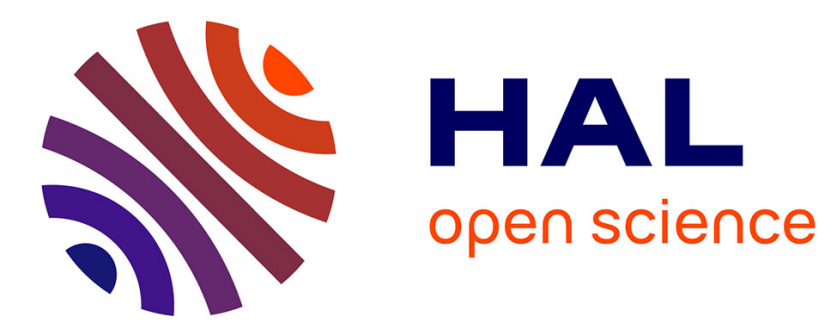

\title{
Control of 2 x 2 Linear Hyperbolic Systems: Backstepping-Based Trajectory Generation and PI-Based Tracking
}

Pierre-Olivier Lamare, Nikolaos Bekiaris-Liberis

\section{- To cite this version:}

Pierre-Olivier Lamare, Nikolaos Bekiaris-Liberis. Control of 2 x 2 Linear Hyperbolic Systems: Backstepping-Based Trajectory Generation and PI-Based Tracking. Systems and Control Letters, 2015, 86, pp.24-33. 10.1016/j.sysconle.2015.09.009 . hal-01242324

HAL Id: hal-01242324

https://hal.inria.fr/hal-01242324

Submitted on 11 Dec 2015

HAL is a multi-disciplinary open access archive for the deposit and dissemination of scientific research documents, whether they are published or not. The documents may come from teaching and research institutions in France or abroad, or from public or private research centers.
L'archive ouverte pluridisciplinaire HAL, est destinée au dépôt et à la diffusion de documents scientifiques de niveau recherche, publiés ou non, émanant des établissements d'enseignement et de recherche français ou étrangers, des laboratoires publics ou privés. 


\title{
Control of $2 \times 2$ Linear Hyperbolic Systems: Backstepping-Based Trajectory Generation and PI-Based Tracking ${ }^{\text {th }}$
}

\author{
Pierre-Olivier Lamare ${ }^{\mathrm{a}, *}$, Nikolaos Bekiaris-Liberis ${ }^{\mathrm{b}}$ \\ ${ }^{a}$ Laboratoire Jean Kuntzmann, Université de Grenoble, BP 53, 38041 Grenoble, France. \\ ${ }^{b}$ Departments of Civil \& Environmental Engineering and Electrical Engineering \& Computer Sciences, University of \\ California, Berkeley, Berkeley, CA 94704, USA.
}

\begin{abstract}
We consider the problems of trajectory generation and tracking for general $2 \times 2$ systems of firstorder linear hyperbolic PDEs with anti-collocated boundary input and output. We solve the trajectory generation problem via backstepping. The reference input, which generates the desired output, incorporates integral operators acting on advanced and delayed versions of the reference output with kernels which were derived by Vazquez, Krstic, and Coron for the backstepping stabilization of $2 \times 2$ linear hyperbolic systems. We apply our approach to a wave PDE with indefinite in-domain and boundary damping. For tracking the desired trajectory we employ a PI control law on the tracking error of the output. We prove exponential stability of the closed-loop system, under the proposed PI control law, when the parameters of the plant and the controller satisfy certain conditions, by constructing a novel "non-diagonal" Lyapunov functional. We demonstrate that the proposed PI control law compensates in the output the effect of in-domain and boundary disturbances. We illustrate our results with numerical examples.
\end{abstract}

Keywords: Distributed parameter systems, Hyperbolic systems, Backstepping, Lyapunov function

\section{Introduction}

Control of $2 \times 2$ systems of first-order hyperbolic PDEs is an active area of research since numerous processes can be modeled with this class of PDE systems. Among various applications, $2 \times 2$ systems model the dynamics of traffic [16], [18], hydraulic [2], [9], [13], [14], as well as gas pipeline networks [19], and the dynamics of transmission lines [8].

Several articles are dedicated to the control and analysis of $2 \times 2$ linear [2], [10], [13], [25] [33], [34], [35] and nonlinear [5], [6], [7], [20], [28], [29] systems. Results for the control of $n \times n$ systems also exist [11], [12], [22], [24]. Algorithms for disturbance rejection in $2 \times 2$ systems are recently developed [1], [32]. The motion planning problem is solved in [15], [26], for a class of $2 \times 2$ systems and in [17], [27] for a class of wave PDEs. Perhaps the most relevant results to the present article are the results in [13], dealing with the Lyapunov-based output-feedback control of

\footnotetext{
This research was partially supported by the LabEx PERSYVAL-Lab (ANR-11-LABX-0025)

*Corresponding author.

Email addresses: pierre-olivier . lamare@imag.fr (Pierre-Olivier Lamare), bekiaris-liberis@berkeley.edu (Nikolaos Bekiaris-Liberis)
} 
$2 \times 2$ linear systems, the results in [34], dealing with the backstepping stabilization of $2 \times 2$ linear systems, and the results in [26], dealing with the motion planning for a class of $2 \times 2$ systems.

In this paper, we are concerned with the trajectory generation and tracking problems for general $2 \times 2$ systems of first-order linear hyperbolic PDEs with anti-collocated boundary input and output. We solve the motion planning problem for this class of systems employing backstepping (Section 2.1). Specifically, we start from a simple transformed system, namely, a cascade of two first-order hyperbolic PDEs, for which the motion planning problem can be trivially solved. We then apply an inverse backstepping transformation to derive the reference trajectory and reference input for the original system. Our approach is different than the one in [34], in that we use backstepping for trajectory generation rather than stabilization, and the one in [26], in that we employ a different conceptual idea to a different class of systems. The idea of the backstepping-based trajectory generation for PDEs, which was conceived in [23], is applied to a beam PDE in [31] and the NavierStokes equations in [4], and is recently extended to general $n \times n$ linear hyperbolic systems in [22]. We apply this methodology to a wave PDE with indefinite in-domain and boundary damping by transforming (see, for example, [3]) the wave PDE to a $2 \times 2$ linear hyperbolic system coupled with a first-order ODE (Section 2.2).

We then employ a PI control law for the stabilization of the error system, namely, the system whose state is defined as the difference between the state of the plant and the reference trajectory. We prove exponential stability in the $L_{2}$ norm of the closed-loop system by constructing a Lyapunov functional which incorporates cross-terms between the PDE states of the system and the ODE state of the controller, when the parameters of the system and the controller satisfy certain conditions (Section 3.1). Our result differs than the result in [13] in that we employ PI control on an output of the system in the Riemann coordinates and we construct a non-diagonal Lyapunov functional for proving closed-loop stability. We demonstrate that the proposed PI control law is capable of compensating in the output the effect of additive disturbances affecting the boundary or the interior of the PDE domain (Section 3.2). We present several examples, for the illustration of our methodologies, including a simulation example dealing with the generation of a sinusoidal reference trajectory for a wave PDE (Section 4.1) and a simulation example of a system tracking a sinusoidal reference output (Section 4.2).

\section{Trajectory Generation Using Backstepping}

\subsection{General $2 \times 2$ Linear Hyperbolic Systems}

We consider the following system

$$
\begin{aligned}
z_{t}^{1}+\varepsilon_{1}(x) z_{x}^{1} & =c_{1}(x) z^{1}+c_{2}(x) z^{2} \\
z_{t}^{2}-\varepsilon_{2}(x) z_{x}^{2} & =c_{3}(x) z^{1}+c_{4}(x) z^{2}
\end{aligned}
$$

under the boundary conditions

$$
\begin{aligned}
z^{1}(0, t) & =q z^{2}(0, t) \\
z^{2}(1, t) & =S(t) \\
z^{2}(0, t) & =y(t)
\end{aligned}
$$


where $t \in[0,+\infty)$ is the time variable, $x \in[0,1]$ is the spatial variable, $y$ is the output of the system, and $S$ is the control input. The functions $\varepsilon_{1}, \varepsilon_{2}$ belong to $C^{2}([0,1])$ and satisfy $\varepsilon_{1}(x), \varepsilon_{2}(x)>0$, for all $x \in[0,1]$, and the functions $c_{i}, i=1,2,3,4$ belong to $C^{1}([0,1])$.

Defining the change of variables (see, for example, [2])

$$
\begin{aligned}
\chi_{1}(x) & =\exp \left(-\int_{0}^{x} \frac{c_{1}(s)}{\varepsilon_{1}(s)} d s\right) \\
\chi_{2}(x) & =\exp \left(\int_{0}^{x} \frac{c_{4}(s)}{\varepsilon_{2}(s)} d s\right) \\
\chi(x) & =\frac{\chi_{1}(x)}{\chi_{2}(x)},
\end{aligned}
$$

and the new coordinates

$$
\begin{aligned}
& u=\chi_{1}(x) z^{1} \\
& v=\chi_{2}(x) z^{2}
\end{aligned}
$$

system (1)-(5) is transformed into the following system

$$
\begin{aligned}
u_{t}+\varepsilon_{1}(x) u_{x} & =\gamma_{1}(x) v \\
v_{t}-\varepsilon_{2}(x) v_{x} & =\gamma_{2}(x) u
\end{aligned}
$$

with

$$
\begin{aligned}
& \gamma_{1}(x)=\chi(x) c_{2}(x) \\
& \gamma_{2}(x)=\chi^{-1}(x) c_{3}(x) .
\end{aligned}
$$

The boundary conditions become

$$
\begin{aligned}
& u(0, t)=q v(0, t) \\
& v(1, t)=U(t) \\
& v(0, t)=y(t),
\end{aligned}
$$

where the original control variable satisfies

$$
U=\chi_{2}(1) S
$$

We aim at designing a reference control input $U^{r}(t)$ such that the output $y(t)$ follows a given reference trajectory $y^{r}(t)$, for $t \geq 0$. For achieving this we need first to construct the reference trajectory $\left(u^{r}(x, t), v^{r}(x, t)\right)$ that satisfies (11), (12), (15), and (17) with $y(t)=y^{r}(t)$. The trajectory generation problem is solvable when the initial data $\left(u^{0}, v^{0}\right)$ match the reference trajectory, i.e., when $u^{0}(x)=u^{r}(x, 0)$ and $v^{0}(x)=v^{r}(x, 0)$ (and hence, the initial data belong to the same space with $u^{r}(x, 0)$ and $\left.v^{r}(x, 0)\right)$. 
Theorem 1. Let $y^{r} \in C^{1}(\mathbb{R})$ be uniformly bounded. The functions

$$
\begin{aligned}
u^{r}(x, t)= & q y^{r}\left(t-\Phi_{1}(x)\right)+\int_{0}^{x} \frac{f(\xi)}{\varepsilon_{1}(\xi)} y^{r}\left(t-\Phi_{1}(x)+\Phi_{1}(\xi)\right) d \xi \\
& +q \int_{0}^{x} L^{\alpha \alpha}(x, \xi) y^{r}\left(t-\Phi_{1}(\xi)\right) d \xi \\
& +\int_{0}^{x} L^{\alpha \alpha}(x, \xi) \int_{0}^{\xi} \frac{f(\zeta)}{\varepsilon_{1}(\zeta)} y^{r}\left(t-\Phi_{1}(\xi)+\Phi_{1}(\zeta)\right) d \zeta d \xi \\
& +\int_{0}^{x} L^{\alpha \beta}(x, \xi) y^{r}\left(t+\Phi_{2}(\xi)\right) d \xi \\
v^{r}(x, t)= & y^{r}\left(t+\Phi_{2}(x)\right)+q \int_{0}^{x} L^{\beta \alpha}(x, \xi) y^{r}\left(t-\Phi_{1}(\xi)\right) d \xi \\
& +\int_{0}^{x} L^{\beta \alpha}(x, \xi) \int_{0}^{\xi} \frac{f(\zeta)}{\varepsilon_{1}(\zeta)} y^{r}\left(t-\Phi_{1}(\xi)+\Phi_{1}(\zeta)\right) d \zeta d \xi \\
& +\int_{0}^{x} L^{\beta \beta}(x, \xi) y^{r}\left(t+\Phi_{2}(\xi)\right) d \xi \\
& y^{r}\left(t+\Phi_{2}(1)\right)+q \int_{0}^{1} L^{\beta \alpha}(1, \xi) y^{r}\left(t-\Phi_{1}(\xi)\right) d \xi \\
& +\int_{0}^{1} L^{\beta \alpha}(1, \xi) \int_{0}^{\xi} \frac{f(\zeta)}{\varepsilon_{1}(\zeta)} y^{r}\left(t-\Phi_{1}(\xi)+\Phi_{1}(\zeta)\right) d \zeta d \xi \\
& +\int_{0}^{1} L^{\beta \beta}(1, \xi) y^{r}\left(t+\Phi_{2}(\xi)\right) d \xi,
\end{aligned}
$$

where

$$
\begin{aligned}
\Phi_{1}(x) & =\int_{0}^{x} \frac{1}{\varepsilon_{1}(s)} d s \\
\Phi_{2}(x) & =\int_{0}^{x} \frac{1}{\varepsilon_{2}(s)} d s \\
f(x) & = \begin{cases}\varepsilon_{2}(0) K^{u v}(x, 0), & \text { if } q=0 \\
0, & \text { if } q \neq 0,\end{cases}
\end{aligned}
$$

and $L^{\alpha \alpha}, L^{\alpha \beta}, L^{\beta \alpha}, L^{\beta \beta}, K^{u v}$ are the solutions of the following equations

$$
\begin{aligned}
& \varepsilon_{2}(x) L_{x}^{\beta \alpha}-\varepsilon_{1}(\xi) L_{\xi}^{\beta \alpha}=\varepsilon_{1}^{\prime}(\xi) L^{\beta \alpha}-\gamma_{2}(x) L^{\alpha \alpha} \\
& \varepsilon_{2}(x) L_{x}^{\beta \beta}+\varepsilon_{2}(\xi) L_{\xi}^{\beta \beta}=-\varepsilon_{2}^{\prime}(\xi) L^{\beta \beta}-\gamma_{2}(x) L^{\alpha \beta} \\
& \varepsilon_{1}(x) L_{x}^{\alpha \alpha}+\varepsilon_{1}(\xi) L_{\xi}^{\alpha \alpha}=-\varepsilon_{1}^{\prime}(\xi) L^{\alpha \alpha}+\gamma_{1}(x) L^{\beta \alpha} \\
& \varepsilon_{1}(x) L_{x}^{\alpha \beta}-\varepsilon_{2}(\xi) L_{\xi}^{\alpha \beta}=\varepsilon_{2}^{\prime}(\xi) L^{\alpha \beta}+\gamma_{1}(x) L^{\beta \beta}
\end{aligned}
$$




$$
\begin{aligned}
& \varepsilon_{1}(x) K_{x}^{u u}+\varepsilon_{1}(\xi) K_{\xi}^{u u}=-\varepsilon_{1}^{\prime}(\xi) K^{u u}-\gamma_{2}(x) K^{u v} \\
& \varepsilon_{1}(x) K_{x}^{u v}-\varepsilon_{2}(\xi) K_{\xi}^{u v}=\varepsilon_{2}^{\prime}(\xi) K^{u v}-\gamma_{1}(x) K^{u u}
\end{aligned}
$$

with the boundary conditions

$$
\begin{aligned}
L^{\beta \alpha}(x, x) & =-\frac{\gamma_{2}(x)}{\varepsilon_{1}(x)+\varepsilon_{2}(x)} \\
L^{\alpha \alpha}(x, 0) & = \begin{cases}h_{1}(x), & \text { if } q=0 \\
\frac{\varepsilon_{2}(0)}{q \varepsilon_{1}(0)} L^{\alpha \beta}(x, 0), & \text { if } q \neq 0\end{cases} \\
L^{\beta \beta}(x, 0) & = \begin{cases}\frac{1}{\varepsilon_{2}(0)} \int_{0}^{x} L^{\beta \alpha}(x, \xi) f(\xi) d \xi, & \text { if } q=0 \\
\frac{q \varepsilon_{1}(0)}{\varepsilon_{2}(0)} L^{\beta \alpha}(x, 0), & \text { if } q \neq 0\end{cases} \\
L^{\alpha \beta}(x, x) & =\frac{\gamma_{1}(x)}{\varepsilon_{1}(x)+\varepsilon_{2}(x)} \\
K^{u u}(x, 0) & =h_{2}(x) \\
K^{u v}(x, x) & =\frac{\gamma_{1}(x)}{\varepsilon_{1}(x)+\varepsilon_{2}(x)},
\end{aligned}
$$

where $h_{1}, h_{2} \in C^{1}([0,1])$ are arbitrary, are uniformly bounded and solve the boundary value problem (11), (12), (15), (16). In particular, $v^{r}(0, t)=y^{r}(t)$, for $t \geq 0$.

Before proving Theorem 1 we make the following observation, which is also helpful in understanding better the proof strategy of Theorem 1 .

Remark 1. The approach for the trajectory generation introduced here is inspired from backstepping. Consider the following system

$$
\begin{array}{r}
\alpha_{t}+\varepsilon_{1}(x) \alpha_{x}-f(x) \beta(0, t)=0 \\
\beta_{t}-\varepsilon_{2}(x) \beta_{x}=0
\end{array}
$$

with boundary condition

$$
\alpha(0, t)=q \beta(0, t)
$$

which follows by directly applying the backstepping transformation

$$
\alpha(x, t)=u(x, t)-\int_{0}^{x} K^{u u}(x, \xi) u(\xi, t) d \xi-\int_{0}^{x} K^{u v}(x, \xi) v(\xi, t) d \xi
$$




$$
\beta(x, t)=v(x, t)-\int_{0}^{x} K^{v u}(x, \xi) u(\xi, t) d \xi-\int_{0}^{x} K^{v v}(x, \xi) v(\xi, t) d \xi
$$

where the kernels $K^{u u}, K^{u v}, K^{v u}, K^{v v}$ are given in [34], to system (11), (12), and (15). It is shown that the functions

$$
\begin{aligned}
& \alpha(x, t)=q y^{r}\left(t-\Phi_{1}(x)\right)+\int_{0}^{x} \frac{f(\xi)}{\varepsilon_{1}(\xi)} y^{r}\left(t-\Phi_{1}(x)+\Phi_{1}(\xi)\right) d \xi \\
& \beta(x, t)=y^{r}\left(t+\Phi_{2}(x)\right),
\end{aligned}
$$

where $\Phi_{1}$ and $\Phi_{2}$ are defined in (22) and (23), respectively, satisfy (37)-(39) with

$$
\beta(1, t)=y^{r}\left(t+\Phi_{2}(1)\right)
$$

and, in particular, $\beta(0, t)=y^{r}(t)$. Using the inverse backstepping transformations introduced in [34]

$$
\begin{aligned}
& u(x, t)=\alpha(x, t)+\int_{0}^{x} L^{\alpha \alpha}(x, \xi) \alpha(\xi, t) d \xi+\int_{0}^{x} L^{\alpha \beta}(x, \xi) \beta(\xi, t) d \xi \\
& v(x, t)=\beta(x, t)+\int_{0}^{x} L^{\beta \alpha}(x, \xi) \alpha(\xi, t) d \xi+\int_{0}^{x} L^{\beta \beta}(x, \xi) \beta(\xi, t) d \xi
\end{aligned}
$$

and relations 42, 43 one can conclude that the functions $u^{r}, v^{r}$, and $U^{r}=v^{r}(1)$ solve the trajectory generation problem for system $(11),(12),(15)-(17)$.

Note that the present approach cannot be directly applied to cases where $\varepsilon_{1}(x)$ or $\varepsilon_{2}(x)$ vanish for some $x \in[0,1]$. This is evident, for instance, from $(33)$ which would imply that the kernel $L^{\beta \beta}$ of the open-loop control law $U^{r}$ may become infinity for all $x \in[0,1]$.

Proof We first consider the case $q \neq 0$. Note that since $\varepsilon_{1}, \varepsilon_{2} \in C^{2}([0,1])$ with $\varepsilon_{1}(x), \varepsilon_{2}(x)>0$, for all $x \in[0,1]$ and $\gamma_{1}, \gamma_{2} \in C^{1}([0,1])$, system $[25)-(34)$ has a unique solution with $L^{\alpha \alpha}, L^{\alpha \beta}, L^{\beta \alpha}$, $L^{\beta \beta} \in C^{1}(\mathscr{T})$ where $\mathscr{T}=\{(x, \xi): 0 \leq \xi \leq x \leq 1\}$ [7]. Hence, from [19]-21] and the uniform boundedness of $y^{r}$ it follows that $u^{r}, v^{r}$, and $U^{r}$ are bounded for all $t \geq 0$ and $x \in[0,1]$.

Taking the time and space derivatives of $u^{r}$ we get

$$
\begin{aligned}
u_{t}^{r}+\varepsilon_{1}(x) u_{x}^{r}= & q \int_{0}^{x} L^{\alpha \alpha}(x, \xi) y^{r^{\prime}}\left(t-\Phi_{1}(\xi)\right) d \xi+\int_{0}^{x} L^{\alpha \beta}(x, \xi) y^{r^{\prime}}\left(t+\Phi_{2}(\xi)\right) d \xi \\
& +\varepsilon_{1}(x) \int_{0}^{x} L_{x}^{\alpha \beta}(x, \xi) y^{r}\left(t+\Phi_{2}(\xi)\right) d \xi+q \varepsilon_{1}(x) \int_{0}^{x} L_{x}^{\alpha \alpha}(x, \xi) y^{r}\left(t-\Phi_{1}(\xi)\right) d \xi \\
& +\varepsilon_{1}(x) L^{\alpha \beta}(x, x) y^{r}\left(t+\Phi_{2}(x)\right)+q \varepsilon_{1}(x) L^{\alpha \alpha}(x, x) y^{r}\left(t-\Phi_{1}(x)\right) .
\end{aligned}
$$


Integrating by parts the first two integrals we get

$$
\begin{aligned}
u_{t}^{r}+\varepsilon_{1}(x) u_{x}^{r}= & q \int_{0}^{x}\left(\varepsilon_{1}(x) L_{x}^{\alpha \alpha}(x, \xi)+\varepsilon_{1}(\xi) L_{\xi}^{\alpha \alpha}(x, \xi)+\varepsilon_{1}^{\prime}(\xi) L^{\alpha \alpha}(x, \xi)\right) y^{r}\left(t-\Phi_{1}(\xi)\right) d \xi \\
& +\int_{0}^{x}\left(\varepsilon_{1}(x) L_{x}^{\alpha \beta}(x, \xi)-\varepsilon_{2}(\xi) L_{\xi}^{\alpha \beta}(x, \xi)-\varepsilon_{2}^{\prime}(\xi) L^{\alpha \beta}(x, \xi)\right) y^{r}\left(t+\Phi_{2}(\xi)\right) d \xi \\
& +\left(q \varepsilon_{1}(0) L^{\alpha \alpha}(x, 0)-\varepsilon_{2}(0) L^{\alpha \beta}(x, 0)\right) y^{r}(t) \\
& +\left(\varepsilon_{1}(x)+\varepsilon_{2}(x)\right) L^{\alpha \beta}(x, x) y^{r}\left(t+\Phi_{2}(x)\right) .
\end{aligned}
$$

Due to the fact that $L^{\alpha \beta}$ and $L^{\alpha \alpha}$ are the solutions of 27 and 28 with the boundary conditions (32) and (34) one gets, by using (20), that $u^{r}$ satisfies (11). The proof that $v^{r}$ satisfies (12) follows analogously. Setting $x=0$ in (19), (20) and using (22), (23), we get that $u^{r}$ and $v^{r}$ satisfy (15). Setting $x=1$ in (20) it follows that (21) satisfies (16). Setting in (20) $x=0$ and using (23) we get $v^{r}(0, t)=y^{r}(t)$.

Let us consider next the case $q=0$. First observe that the PDEs (25), (27) with boundary conditions (31), (32), for the kernels $L^{\alpha \alpha}$ and $L^{\beta \alpha}$ are decoupled, and hence, $L^{\alpha \alpha}$ and $L^{\beta \alpha}$ are well-defined [7]. Hence, since $f$ satisfies (24) and $K^{u v}, K^{u u}$ are well-defined [7], one can conclude that $L^{\alpha \beta}$ and $L^{\beta \beta}$ are well-defined as well.

Taking the time and space derivatives of $u^{r}$ we get

$$
\begin{aligned}
u_{t}^{r}+\varepsilon_{1}(x) u_{x}^{r}= & f(x) y^{r}(t)+\int_{0}^{x} L^{\alpha \alpha}(x, \xi) \int_{0}^{\xi} \frac{f(\zeta)}{\varepsilon_{1}(\zeta)} y^{r^{\prime}}\left(t-\Phi_{1}(\xi)+\Phi_{1}(\zeta)\right) d \zeta d \xi \\
& +\int_{0}^{x} L^{\alpha \beta}(x, \xi) y^{r^{\prime}}\left(t+\Phi_{2}(\xi)\right) d \xi \\
& +\varepsilon_{1}(x) L^{\alpha \alpha}(x, x) \int_{0}^{x} \frac{f(\zeta)}{\varepsilon_{1}(\zeta)} y^{r}\left(t-\Phi_{1}(x)+\Phi_{1}(\zeta)\right) d \zeta \\
& +\varepsilon_{1}(x) L^{\alpha \beta}(x, x) y^{r}\left(t+\Phi_{2}(x)\right)+\varepsilon_{1}(x) \int_{0}^{x} L_{x}^{\alpha \beta}(x, \xi) y^{r}\left(t+\Phi_{2}(\xi)\right) d \xi \\
& +\varepsilon_{1}(x) \int_{0}^{x} L_{x}^{\alpha \alpha}(x, \xi) \int_{0}^{\xi} \frac{f(\zeta)}{\varepsilon_{1}(\zeta)} y^{r}\left(t-\Phi_{1}(\xi)+\Phi_{1}(\zeta)\right) d \zeta d \xi
\end{aligned}
$$

Integrating by parts the first two integrals we get

$$
\begin{aligned}
u_{t}^{r}+\varepsilon_{1}(x) u_{x}^{r}= & \int_{0}^{x}\left(\varepsilon_{1}(x) L_{x}^{\alpha \alpha}(x, \xi)+\varepsilon_{1}(\xi) L_{\xi}^{\alpha \alpha}(x, \xi)+\varepsilon_{1}^{\prime}(\xi) L^{\alpha \alpha}(x, \xi)\right) \\
& \times \int_{0}^{\xi} \frac{f(\zeta)}{\varepsilon_{1}(\zeta)} y^{r}\left(t-\Phi_{1}(\xi)+\Phi_{1}(\zeta)\right) d \zeta d \xi \\
& +\int_{0}^{x}\left(\varepsilon_{1}(x) L_{x}^{\alpha \beta}(x, \xi)-\varepsilon_{2}(\xi) L_{\xi}^{\alpha \beta}(x, \xi)-\varepsilon_{2}^{\prime}(\xi) L^{\alpha \beta}(x, \xi)\right) y^{r}\left(t+\Phi_{2}(\xi)\right) d \xi \\
& +\left(\varepsilon_{1}(x)+\varepsilon_{2}(x)\right) L^{\alpha \beta}(x, x) y^{r}\left(t+\Phi_{2}(x)\right) \\
& +y^{r}(t)\left(f(x)+\int_{0}^{x} L^{\alpha \alpha}(x, \xi) f(\xi) d \xi-\varepsilon_{2}(0) L^{\alpha \beta}(x, 0)\right) .
\end{aligned}
$$


Using (20), 27), 28), and (34) one can conclude that $u^{r}$ satisfies (11) if $f$ satisfies

$$
f(x)=\varepsilon_{2}(0) L^{\alpha \beta}(x, 0)-\int_{0}^{x} L^{\alpha \alpha}(x, \xi) f(\xi) d \xi .
$$

This fact can been shown as follows. The inverse of the backstepping transformation (40), (41) is uniquely defined and has the form (45), (46) (see, for example, [21]). Hence, substituting (40]), (41) in (45), (46) we get

$$
\begin{aligned}
& \int_{0}^{x}\left(K^{u u}(x, \xi)-L^{\alpha \alpha}(x, \xi)\right) u(\xi, t)+\left(K^{u v}(x, \xi)-L^{\alpha \beta}(x, \xi)\right) v(\xi, t) d \xi \\
& +\int_{0}^{x} \int_{0}^{\xi}\left(\left(L^{\alpha \alpha}(x, \xi) K^{u u}(\xi, \zeta)+L^{\alpha \beta}(x, \xi) K^{v u}(\xi, \zeta)\right) u(\zeta, t)\right. \\
& \left.+\left(L^{\alpha \alpha}(x, \xi) K^{u v}(\xi, \zeta)+L^{\alpha \beta}(x, \xi) K^{v v}(\xi, \zeta)\right) v(\zeta, t)\right) d \zeta d \xi=0 .
\end{aligned}
$$

Performing a change in the order of integration in the second integral of (52) and using the fact that (52) holds for all $u$ and $v$, one obtains

$$
K^{u v}(x, \xi)=L^{\alpha \beta}(x, \xi)-\int_{\xi}^{x}\left(L^{\alpha \alpha}(x, s) K^{u v}(s, \xi)+L^{\alpha \beta}(x, s) K^{v v}(s, \xi)\right) d s .
$$

Setting $\xi=0$ in (53), multiplying (53) by $\varepsilon_{2}(0)$, and using the facts that $K^{v v}(x, 0)=0$ for all $x \in[0,1]$ (see relation (31) in [34]) and that $f$ is defined by (24), we get that $f$ satisfies (51) for $q=0$. The rest of the proof is similar to the case $q \neq 0$.

Example 1. We consider the following system

$$
\begin{aligned}
z_{t}^{1}+\varepsilon_{1} z_{x}^{1} & =-\frac{1}{\tau} z^{1} \\
z_{t}^{2}-\varepsilon_{2} z_{x}^{2} & =-\frac{1}{\tau} z^{1},
\end{aligned}
$$

with boundary conditions

$$
\begin{aligned}
& z^{1}(0, t)=q z^{2}(0, t) \\
& z^{2}(1, t)=S(t),
\end{aligned}
$$

where $\tau$ is a positive parameter. Among various systems that can be modeled by (54)-(57) (for instance, the Saint-Venant equations, see [13], [9]), system (54)-(57) can be viewed as a linearized version of the Aw-Rascle-Zhang (ARZ) macroscopic model of traffic flow in the Riemann coordi- 
nates

$$
\begin{aligned}
& z^{1}=w-V^{\prime}\left(s^{*}\right) s \\
& z^{2}=w,
\end{aligned}
$$

where $w$ and $s$ correspond to the velocity and density of the vehicles at time $t$ and location $x$, respectively. The variable $V\left(s^{*}\right)$ is the nominal velocity of the cars and $s^{*}$ is the nominal density. The opposite transport velocities in $(54),(55)$ correspond to traffic flow in a congested mode. The parameter $\frac{1}{\tau}$ is an indicator of the convergence rate of the velocity $w$ of the cars to the nominal velocity $V(s)$. For more details the reader is referred to [16]. The boundary condition [56 in the original variables is written as

$$
w=\frac{V^{\prime}\left(s^{*}\right) s}{1-q} .
$$

Hence, the boundary condition (56) dictates that there is a static relation, at the entrance of the road, between the density and the velocity similarly to the static relation between the nominal velocity $V(s)$ and the density of the cars in the road. The change of variables $(9),(10),(13)$, and $(14)$ transform system (54)-(57) to

$$
\begin{aligned}
u_{t}+\varepsilon_{1} u_{x} & =0 \\
v_{t}-\varepsilon_{2} v_{x} & =-\frac{1}{\tau} \exp \left(-\frac{1}{\tau \varepsilon_{1}} x\right) u \\
u(0, t) & =q v(0, t) \\
v(1, t) & =U(t),
\end{aligned}
$$

where $U(t)$ is given by (18). Observing that $\gamma_{1}=0$, relations $(25)-(34)$ can be solved explicitly as

$$
\begin{aligned}
L^{\alpha \alpha}(x, \xi) & =0 \\
L^{\alpha \beta}(x, \xi) & =0 \\
L^{\beta \alpha}(x, \xi) & =\frac{1}{\tau\left(\varepsilon_{1}+\varepsilon_{2}\right)} \exp \left(-\frac{1}{\tau \varepsilon_{1}}\left(\frac{\varepsilon_{1} x+\varepsilon_{2} \xi}{\varepsilon_{1}+\varepsilon_{2}}\right)\right) \\
L^{\beta \beta}(x, \xi) & =\frac{q \varepsilon_{1}}{\tau \varepsilon_{2}\left(\varepsilon_{1}+\varepsilon_{2}\right)} \exp \left(-\frac{1}{\tau \varepsilon_{1}}\left(\frac{\varepsilon_{1} x-\varepsilon_{1} \xi}{\varepsilon_{1}+\varepsilon_{2}}\right)\right) .
\end{aligned}
$$


Therefore, for system (54)-(57), the reference input which generates the desired output $y^{r}(t)$ is

$$
\begin{aligned}
S^{r}(t)=y^{r} & \left(t+\frac{1}{\varepsilon_{2}}\right)+\frac{q}{\tau\left(\varepsilon_{1}+\varepsilon_{2}\right)} \int_{0}^{1} \exp \left(-\frac{1}{\tau \varepsilon_{1}}\left(\frac{\varepsilon_{1}+\varepsilon_{2} \xi}{\varepsilon_{1}+\varepsilon_{2}}\right)\right) y^{r}\left(t-\frac{\xi}{\varepsilon_{1}}\right) d \xi \\
& +\frac{q \varepsilon_{1}}{\tau \varepsilon_{2}\left(\varepsilon_{1}+\varepsilon_{2}\right)} \int_{0}^{1} \exp \left(-\frac{1}{\tau \varepsilon_{1}}\left(\frac{\varepsilon_{1}-\varepsilon_{1} \xi}{\varepsilon_{1}+\varepsilon_{2}}\right)\right) y^{r}\left(t+\frac{\xi}{\varepsilon_{2}}\right) d \xi
\end{aligned}
$$

\subsection{Application to a Wave PDE with Indefinite In-Domain and Boundary Damping}

Let us consider system

$$
\begin{aligned}
z_{t t} & =\varepsilon(x) z_{x x}+h(x) z_{t}+b(x) z_{x} \\
z_{x}(0, t) & =-g z_{t}(0, t) \\
z_{x}(1, t) & =W(t)
\end{aligned}
$$

with $g \neq\left\{\frac{1}{\sqrt{\varepsilon(0)}},-\frac{1}{\sqrt{\varepsilon(0)}}\right\}, h, b \in C^{1}([0,1])$, and $\varepsilon \in C^{2}([0,1])$ with $\varepsilon(x)>0$, for all $x \in[0,1]$. The objective is $z(0, t)$ to track a reference trajectory, say, $\zeta(t)$, which belongs to $C^{2}(\mathbb{R})$. Let us define the output of the system as

$$
\psi(t)=z(0, t)
$$

With the change of variables

$$
\begin{aligned}
z^{1}(x, t) & =\frac{1-\sqrt{\varepsilon(0)} g}{1+\sqrt{\varepsilon(0)} g}\left(z_{t}(x, t)-\sqrt{\varepsilon(x)} z_{x}(x, t)\right) \\
z^{2}(x, t) & =z_{t}(x, t)+\sqrt{\varepsilon(x)} z_{x}(x, t) \\
S(t) & =\sqrt{\varepsilon(1)} W(t)+z_{t}(1, t),
\end{aligned}
$$

system (70)-(72) is rewritten as (1)-(5) where

$$
\begin{aligned}
y(t) & =(1-\sqrt{\varepsilon(0) g}) \dot{\psi}(t) \\
\varepsilon_{1}(x) & =\sqrt{\varepsilon(x)} \\
\varepsilon_{2}(x) & =\sqrt{\varepsilon(x)} \\
q & =1 \\
c_{1}(x) & =\frac{h(x)}{2}-\frac{b(x)}{2 \sqrt{\varepsilon(x)}}+\frac{\varepsilon^{\prime}(x)}{4 \sqrt{\varepsilon(x)}} \\
c_{2}(x) & =m c_{4}(x) \\
c_{3}(x) & =\frac{1}{m} c_{1}(x) \\
c_{4}(x) & =\frac{h(x)}{2}+\frac{b(x)}{2 \sqrt{\varepsilon(x)}}-\frac{\varepsilon^{\prime}(x)}{4 \sqrt{\varepsilon(x)}}
\end{aligned}
$$




$$
m=\frac{1-\sqrt{\varepsilon(0)} g}{1+\sqrt{\varepsilon(0) g}}
$$

together with the integrator $\dot{\psi}(t)=\frac{1}{1-\sqrt{\varepsilon(0)} g} z^{2}(0, t)$. Applying Theorem 1 we get the following reference input

$$
\begin{aligned}
W^{r}(t)= & \frac{1}{2 \sqrt{\varepsilon(1)}}\left(( 1 - \sqrt { \varepsilon ( 0 ) } g ) \operatorname { e x p } ( - \int _ { 0 } ^ { 1 } \frac { c _ { 4 } ( s ) } { \varepsilon _ { 2 } ( s ) } d s ) \left(\dot{\zeta}\left(t+\Phi_{2}(1)\right)\right.\right. \\
& \left.+\int_{0}^{1} L^{\beta \alpha}(1, \xi) \dot{\zeta}\left(t-\Phi_{1}(\xi)\right) d \xi+\int_{0}^{1} L^{\beta \beta}(1, \xi) \dot{\zeta}\left(t+\Phi_{2}(\xi)\right) d \xi\right) \\
& -(1+\sqrt{\varepsilon(0)} g) \exp \left(\int_{0}^{1} \frac{c_{1}(s)}{\varepsilon_{1}(s)} d s\right)\left(\dot{\zeta}\left(t-\Phi_{1}(1)\right)\right. \\
& \left.\left.+\int_{0}^{1} L^{\alpha \alpha}(x, \xi) \dot{\zeta}\left(t-\Phi_{1}(\xi)\right) d \xi+\int_{0}^{1} L^{\alpha \beta}(x, \xi) \dot{\zeta}\left(t+\Phi_{2}(\xi)\right) d \xi\right)\right) .
\end{aligned}
$$

\section{Trajectory Tracking Using PI Control}

\subsection{Stability Analysis with a Non-Diagonal Lyapunov Functional}

For stabilizing the system around the desired trajectory for any initial condition $(u(x, 0), v(x, 0))$, rather than only for $(u(x, 0), v(x, 0))=\left(u^{r}(x, 0), v^{r}(x, 0)\right)$, we employ a PI-feedback control law. We first write the dynamics of the tracking errors $\tilde{u}(x, t)=u(x, t)-u^{r}(x, t)$ and $\tilde{v}(x, t)=v(x, t)-v^{r}(x, t)$ as

$$
\begin{aligned}
\tilde{u}_{t}+\varepsilon_{1}(x) \tilde{u}_{x} & =\gamma_{1}(x) \tilde{v} \\
\tilde{v}_{t}-\varepsilon_{2}(x) \tilde{v}_{x} & =\gamma_{2}(x) \tilde{u} \\
\tilde{u}(0, t) & =q \tilde{v}(0, t) \\
\tilde{v}(1, t) & =\tilde{U}(t),
\end{aligned}
$$

where $\tilde{U}=U-U^{r}$ and $U^{r}$ is the reference input generating the desired reference trajectory. We employ the controller

$$
\tilde{U}(t)=-k_{P} \tilde{v}(0, t)-k_{I} \tilde{\eta}(t),
$$

with

$$
\dot{\tilde{\eta}}(t)=\tilde{v}(0, t) .
$$

Theorem 2. Consider system (87)-(90) together with the control law (91), 92). Let the positive constants $\mu, \beta, \rho, \gamma, \nu, \kappa$, and $\theta$ be such that the matrices

$$
M_{1}=\left[\begin{array}{cc}
-q^{2}-\beta\left(k_{P}^{2} e^{\mu}-1\right)-\frac{\kappa \gamma}{2} & -\beta k_{P} k_{I} e^{\mu}+\frac{\gamma}{2}\left(e^{v} k_{P}+1\right)-\frac{\rho}{2} \\
-\beta k_{P} k_{I} e^{\mu}+\frac{\gamma}{2}\left(e^{v} k_{P}+1\right)-\frac{\rho}{2} & -\beta k_{I}^{2} e^{\mu}+\gamma e^{v} k_{I}-\frac{\gamma}{2}
\end{array}\right]
$$




$$
M_{2}(x)=\left[\begin{array}{ll}
M_{21}(x) & M_{22}(x) \\
M_{23}(x) & M_{24}(x)
\end{array}\right]
$$

with

$$
\begin{aligned}
& M_{21}(x)=\left(\mu-\frac{\theta}{\varepsilon_{1}(x)}\right) e^{-\mu x}+\frac{\gamma^{2}}{2(\theta \rho-\gamma)} \frac{\gamma_{2}^{2}(x)}{\varepsilon_{2}^{2}(x)} e^{2 v x} \\
& M_{22}(x)=-\frac{\gamma_{1}(x)}{\varepsilon_{1}(x)} e^{-\mu x}-\beta \frac{\gamma_{2}(x)}{\varepsilon_{2}(x)} e^{\mu x}-\frac{\gamma^{2}}{2(\theta \rho-\gamma)} \frac{\gamma_{2}(x)}{\varepsilon_{2}(x)}\left(v-\frac{\theta}{\varepsilon_{2}(x)}\right) e^{2 v x} \\
& M_{23}(x)=-\frac{\gamma_{1}(x)}{\varepsilon_{1}(x)} e^{-\mu x}-\beta \frac{\gamma_{2}(x)}{\varepsilon_{2}(x)} e^{\mu x}-\frac{\gamma^{2}}{2(\theta \rho-\gamma)} \frac{\gamma_{2}(x)}{\varepsilon_{2}(x)}\left(v-\frac{\theta}{\varepsilon_{2}(x)}\right) e^{2 v x} \\
& M_{24}(x)=\beta\left(\mu-\frac{\theta}{\varepsilon_{2}(x)}\right) e^{\mu x}-\frac{\gamma}{2 \kappa} \frac{e^{2 v x}}{\varepsilon_{2}^{2}(x)}+\frac{\gamma^{2}}{2(\theta \rho-\gamma)}\left(v-\frac{\theta}{\varepsilon_{2}(x)}\right)^{2} e^{2 v x},
\end{aligned}
$$

are positive semi-definite for all $x \in[0,1]$, and the inequalities

$$
\begin{aligned}
\beta \rho & >\frac{\gamma^{2} e^{(2 v-\mu) x}}{2 \varepsilon_{2}(x)}, \quad \forall x \in[0,1] \\
\gamma & >\theta \rho
\end{aligned}
$$

hold. Then, there exist positive constants $\lambda$ and $\Omega$ such that, for all initial conditions satisfying $\left(\tilde{u}^{0}(x), \tilde{v}^{0}(x), \tilde{\eta}^{0}\right) \in L^{2}(0,1) \times L^{2}(0,1) \times \mathbb{R}$, the following holds for all $t \geq 0$

$$
\int_{0}^{1}\left(\tilde{u}^{2}(x, t)+\tilde{v}^{2}(x, t)\right) d x+\tilde{\eta}^{2}(t) \leq \Omega e^{-\lambda t}\left(\int_{0}^{1}\left(\tilde{u}^{2}(x, 0)+\tilde{v}^{2}(x, 0)\right) d x+\tilde{\eta}^{2}(0)\right) .
$$

Proof In order to analyze the stability of system (87)- 92 we propose the following Lyapunov functional

$$
\begin{aligned}
V(t) & =\int_{0}^{1}\left[\begin{array}{c}
\tilde{u}(x, t) \\
\tilde{v}(x, t) \\
\tilde{\eta}(t)
\end{array}\right]^{\top} P(x)\left[\begin{array}{c}
\tilde{u}(x, t) \\
\tilde{v}(x, t) \\
\tilde{\eta}(t)
\end{array}\right] d x \\
& =R_{1}(t)+R_{2}(t)+R_{3}(t)+R_{4}(t),
\end{aligned}
$$

with

$$
P(x)=\left[\begin{array}{ccc}
\frac{e^{-\mu x}}{\varepsilon_{1}(x)} & 0 & 0 \\
0 & \beta \frac{e^{\mu x}}{\varepsilon_{2}(x)} & \frac{\gamma e^{v x}}{2 \varepsilon_{2}(x)} \\
0 & \frac{\gamma e^{v x}}{2 \varepsilon_{2}(x)} & \frac{\rho}{2}
\end{array}\right],
$$


and

$$
\begin{aligned}
& R_{1}(t)=\int_{0}^{1} \tilde{u}^{2}(x, t) \frac{e^{-\mu x}}{\varepsilon_{1}(x)} d x \\
& R_{2}(t)=\beta \int_{0}^{1} \tilde{v}^{2}(x, t) \frac{e^{\mu x}}{\varepsilon_{2}(x)} d x \\
& R_{3}(t)=\gamma \tilde{\eta}(t) \int_{0}^{1} \tilde{v}(x, t) \frac{e^{v x}}{\varepsilon_{2}(x)} d x \\
& R_{4}(t)=\frac{\rho}{2} \tilde{\eta}^{2}(t) .
\end{aligned}
$$

Let us introduce the constants

$$
\begin{aligned}
& \underline{\lambda}=\min _{x \in[0,1]} \lambda_{\min }(P(x)) \\
& \bar{\lambda}=\max _{x \in[0,1]} \lambda_{\max }(P(x)) .
\end{aligned}
$$

Inequality (99) ensures that $P(x)$ is positive definite and symmetric for all $x \in[0,1]$, and hence, using the fact that $\varepsilon_{1}, \varepsilon_{2} \in C^{2}([0,1])$ with $\varepsilon_{1}(x), \varepsilon_{2}(x)>0$, for all $x \in[0,1]$, one can conclude that, $\bar{\lambda}, \underline{\lambda}>0$. Therefore,

$$
\underline{\lambda}\left(\int_{0}^{1}\left(\tilde{u}^{2}(x, t)+\tilde{v}^{2}(x, t)\right) d x+\tilde{\eta}^{2}(t)\right) \leq V(t) \leq \bar{\lambda}\left(\int_{0}^{1}\left(\tilde{u}^{2}(x, t)+\tilde{v}^{2}(x, t)\right) d x+\tilde{\eta}^{2}(t)\right) .
$$

Using (104)-107) we get along the solutions of system (87)-92 that

$$
\begin{aligned}
\dot{R}_{1}(t)= & -2 \int_{0}^{1} \tilde{u}(x, t) \tilde{u}_{x}(x, t) e^{-\mu x} d x+2 \int_{0}^{1} \tilde{u}(x, t) \tilde{v}(x, t) \frac{\gamma_{1}(x)}{\varepsilon_{1}(x)} e^{-\mu x} d x \\
= & \left(q^{2} \tilde{v}^{2}(0, t)-e^{-\mu} \tilde{u}^{2}(1, t)\right)-\mu \int_{0}^{1} \tilde{u}^{2}(x, t) e^{-\mu x} d x+2 \int_{0}^{1} \tilde{u}(x, t) \tilde{v}(x, t) \frac{\gamma_{1}(x)}{\varepsilon_{1}(x)} e^{-\mu x} d x \\
\dot{R}_{2}(t)= & 2 \beta \int_{0}^{1} \tilde{v}(x, t) \tilde{v}_{x}(x, t) e^{\mu x} d x+2 \beta \int_{0}^{1} \tilde{u}(x, t) \tilde{v}(x, t) \frac{\gamma_{2}(x)}{\varepsilon_{2}(x)} e^{\mu x} d x \\
= & \beta\left(k_{P}^{2} e^{\mu} \tilde{v}^{2}(0, t)+2 k_{P} k_{I} e^{\mu} \tilde{v}(0, t) \tilde{\eta}(t)+k_{I}^{2} e^{\mu} \tilde{\eta}^{2}(t)-\tilde{v}^{2}(0, t)\right)-\mu \beta \int_{0}^{1} \tilde{v}^{2}(x, t) e^{\mu x} d x \\
& +2 \beta \int_{0}^{1} \tilde{u}(x, t) \tilde{v}(x, t) \frac{\gamma_{2}(x)}{\varepsilon_{2}(x)} e^{\mu x} d x \\
\dot{R}_{3}(t)= & \gamma \tilde{\eta}(t) \int_{0}^{1} \tilde{v}_{x}(x, t) e^{v x} d x+\gamma \tilde{v}(0, t) \int_{0}^{1} \tilde{v}(x, t) \frac{e^{v x}}{\varepsilon_{2}(x)} d x+\gamma \tilde{\eta}(t) \int_{0}^{1} \tilde{u}(x, t) \frac{\gamma_{2}(x)}{\varepsilon_{2}(x)} e^{v x} d x \\
\leq & \gamma \tilde{\eta}(t)\left(e^{v}\left(-k_{P} \tilde{v}(0, t)-k_{I} \tilde{\eta}(t)\right)-\tilde{v}(0, t)\right)-v \gamma \tilde{\eta}(t) \int_{0}^{1} \tilde{v}(x, t) e^{v x} d x \\
& +\frac{\kappa \gamma}{2} \tilde{v}^{2}(0, t)+\frac{\gamma}{2 \kappa} \int_{0}^{1} \tilde{v}^{2}(x, t) \frac{e^{2 v x}}{\varepsilon_{2}^{2}(x)} d x+\gamma \tilde{\eta}(t) \int_{0}^{1} \tilde{u}(x, t) \frac{\gamma_{2}(x)}{\varepsilon_{2}(x)} e^{v x} d x \\
\dot{R}_{4}(t)= & \rho \tilde{v}(0, t) \tilde{\eta}(t),
\end{aligned}
$$


where we used integration by parts in the first terms of (111)- 113) and Young's inequality in the second term of (113). Using (102), (111)-(114) we get

$$
\begin{aligned}
\dot{V}(t) \leq & -\left[\begin{array}{c}
\tilde{v}(0, t) \\
\tilde{\eta}(t)
\end{array}\right]^{\top} M_{1}\left[\begin{array}{c}
\tilde{v}(0, t) \\
\tilde{\eta}(t)
\end{array}\right]-\int_{0}^{1}\left[\begin{array}{c}
\tilde{u}(x, t) \\
\tilde{v}(x, t) \\
\tilde{\eta}(t)
\end{array}\right]^{\top} M(x)\left[\begin{array}{c}
\tilde{u}(x, t) \\
\tilde{v}(x, t) \\
\tilde{\eta}(t)
\end{array}\right] d x \\
& -e^{-\mu} \tilde{u}^{2}(1, t)-\theta V(t)
\end{aligned}
$$

where $M_{1}$ is given by $(93)$ and

$$
M(x)=\left[\begin{array}{cc}
A(x) & B^{\top}(x) \\
B(x) & C
\end{array}\right]
$$

with

$$
A(x)=\left[\begin{array}{ll}
A_{1}(x) & A_{2}(x) \\
A_{3}(x) & A_{4}(x)
\end{array}\right],
$$

where

$$
\begin{aligned}
A_{1}(x) & =\left(\mu-\frac{\theta}{\varepsilon_{1}(x)}\right) e^{-\mu x} \\
A_{2}(x) & =-\frac{\gamma_{1}(x)}{\varepsilon_{1}(x)} e^{-\mu x}-\beta \frac{\gamma_{2}(x)}{\varepsilon_{2}(x)} e^{\mu x} \\
A_{3}(x) & =-\frac{\gamma_{1}(x)}{\varepsilon_{1}(x)} e^{-\mu x}-\beta \frac{\gamma_{2}(x)}{\varepsilon_{2}(x)} e^{\mu x} \\
A_{4}(x) & =\beta\left(\mu-\frac{\theta}{\varepsilon_{2}(x)}\right) e^{\mu x}-\frac{\gamma}{2 \kappa} \frac{e^{2 v x}}{\varepsilon_{2}^{2}(x)} \\
B(x) & =\left[-\frac{\gamma}{2} \frac{\gamma_{2}(x)}{\varepsilon_{2}(x)} e^{v x} \frac{\gamma}{2}\left(v-\frac{\theta}{\varepsilon_{2}(x)}\right) e^{v x}\right] \\
C & =\frac{\gamma-\theta \rho}{2} .
\end{aligned}
$$

Using the Schur complement of $C$ in $M(x)$ and $(100,, 123)$ one has that $M(x) \geq 0$ for all $x \in[0,1]$, if and only if

$$
M_{2}(x)=A(x)-B^{\top}(x) C^{-1} B(x) \geq 0 .
$$

Thus, if $M_{1} \geq 0$ and $M_{2}(x) \geq 0$, for all $x \in[0,1]$, one has

$$
\dot{V}(t) \leq-e^{-\mu} \tilde{u}^{2}(1, t)-\theta V(t),
$$

and hence, $V(t) \leq e^{-\theta t} V(0)$, for all $t \geq 0$. Combining this relation with 110 the proof is complete.

Remark 2. A control law with an integral action is designed in [13] for $2 \times 2$ hyperbolic systems. Stability of the closed-loop system is proved using a diagonal Lyapunov functional. Here the nondiagonal term in the Lyapunov functional is needed for proving stability using a quadratic Lyapunov 
functional. Indeed, let us assume that the Lyapunov functional is diagonal. We can write it as

$$
V(t)=\int_{0}^{1}\left(q_{1}(x) \tilde{u}^{2}(x, t)+q_{2}(x) \tilde{v}^{2}(x, t)\right) d x+\frac{\rho}{2} \tilde{\eta}^{2}(t)
$$

where the functions $q_{1}$ and $q_{2}$ belong to $C^{1}([0,1])$ with $q_{1}(x), q_{2}(x)>0$, for all $x \in[0,1]$. The time derivative of $V$ along the solutions of system (87), (88) with boundary conditions $89,-(92)$ is given by

$$
\begin{aligned}
\dot{V}(t)= & {\left[\begin{array}{c}
\tilde{v}(0, t) \\
\tilde{\eta}(t)
\end{array}\right]^{\top}\left[\begin{array}{cc}
D_{1} & D_{2} \\
D_{3} & D_{4}
\end{array}\right]\left[\begin{array}{c}
\tilde{v}(0, t) \\
\tilde{\eta}(t)
\end{array}\right]+\int_{0}^{1}\left[\begin{array}{c}
\tilde{u}(x, t) \\
\tilde{v}(x, t)
\end{array}\right]^{\top} E(x)\left[\begin{array}{c}
\tilde{u}(x, t) \\
\tilde{v}(x, t)
\end{array}\right] d x } \\
& -q_{1}(1) \varepsilon_{1}(1) \tilde{u}^{2}(1, t),
\end{aligned}
$$

where

$$
\begin{aligned}
D_{1} & =q_{1}(0) \varepsilon_{1}(0) q^{2}-q_{2}(0) \varepsilon_{2}(0)+q_{2}(1) \varepsilon_{2}(1) k_{P}^{2} \\
D_{2} & =\frac{1}{2}\left(q_{2}(1) \varepsilon_{2}(1) k_{P} k_{I}+\rho\right) \\
D_{3} & =\frac{1}{2}\left(q_{2}(1) \varepsilon_{2}(1) k_{P} k_{I}+\rho\right) \\
D_{4} & =q_{2}(1) \varepsilon_{2}(1) k_{I}^{2} \\
E(x) & =\left[\begin{array}{cc}
\left(q_{1}(x) \varepsilon_{1}(x)\right)_{x} & q_{1}(x) \gamma_{1}(x)+q_{2}(x) \gamma_{2}(x) \\
q_{1}(x) \gamma_{1}(x)+q_{2}(x) \gamma_{2}(x) & -\left(q_{2}(x) \varepsilon_{2}(x)\right)_{x}
\end{array}\right]
\end{aligned}
$$

Using $(127)$ and 131 one can conclude that when $k_{I} \neq 0$ the inequality $\dot{V} \leq 0$ can not be satisfied for any $\left[\begin{array}{ccc}\tilde{u} & \tilde{v} & \tilde{\eta}\end{array}\right]^{\top}$.

In [2], it is proved that if there exist two boundary controllers for $2 \times 2$ linear hyperbolic systems of the form 87, , 88 such that the functional

$$
V(t)=\int_{0}^{1} q_{1}(x) \tilde{u}^{2}(x, t)+q_{2}(x) \tilde{v}^{2}(x, t)+q_{3}(x) \tilde{u}(x, t) \tilde{v}(x, t) d x
$$

along the solutions of the system (87), (88) with the state $(\tilde{u}, \tilde{v})$ satisfies $\dot{V}<0$ then the cross term $q_{3}$ between $\tilde{u}$ and $\tilde{v}$ is necessarily identically zero. However, in the case of stabilization of $2 \times 2$ linear hyperbolic systems of the form (87), 88) with a PI control law that we consider here, the 
cross term (106) in the Lyapunov functional (102) between the integral state $\tilde{\eta}$ of the controller and the state of the plant $\tilde{v}$ is necessary (as explained above) for proving stability of the overall closed-loop system consisting of the plant state $(\tilde{u}, \tilde{v})$ and the integral state $\tilde{\eta}$, using the Lyapunov functional defined in (102) (although a cross term between $\tilde{u}$ and $\tilde{v}$ is not necessary).

As explained in Remark 2 the non-diagonal term in the Lyapunov functional is crucial for proving stability using a quadratic Lyapunov functional. However, this term adds considerable complexity in verifying analytically that the matrices (93), (94) are positive definite and that (99) holds. In general, the positivity of $M_{1}$ and $M_{2}$ must be verified numerically. Yet, from the expression of $M_{1}$ we see that a necessary condition is that $k_{I}$ is strictly positive. In addition, from (93) it is evident that $k_{P}$ must satisfy $\left|k_{P}\right|<1$. Note that from (93)-(100) it seems possible that the positivity of $M_{1}$ and $M_{2}$ may depend on the values of $\gamma_{1}, \gamma_{2}$, and $q$. Next, we numerically verify the conditions of Theorem 2 for the system from Example 1.

Example 2 (Example 11Continued). We set in 61-63

$$
\begin{aligned}
\varepsilon_{1} & =3 \\
\varepsilon_{2} & =6 \\
\tau & =5 \\
q & =0.2,
\end{aligned}
$$

and choose $U$ in 90 according to (91) with

$$
\begin{aligned}
& k_{P}=0.1 \\
& k_{I}=1.0583,
\end{aligned}
$$

in order to stabilize the zero equilibrium of $61-63$. We verify numerically that the conditions of Theorem 2 are satisfied with

$$
(\beta, \kappa, \mu, \nu, \theta, \rho, \gamma)=(0.7,0.2,0.5,0.2,0.7,2,2)
$$

From 93 we get that

$$
M_{1}=\left[\begin{array}{cc}
0.4485 & 0 \\
0 & 0.2926
\end{array}\right]>0 .
$$




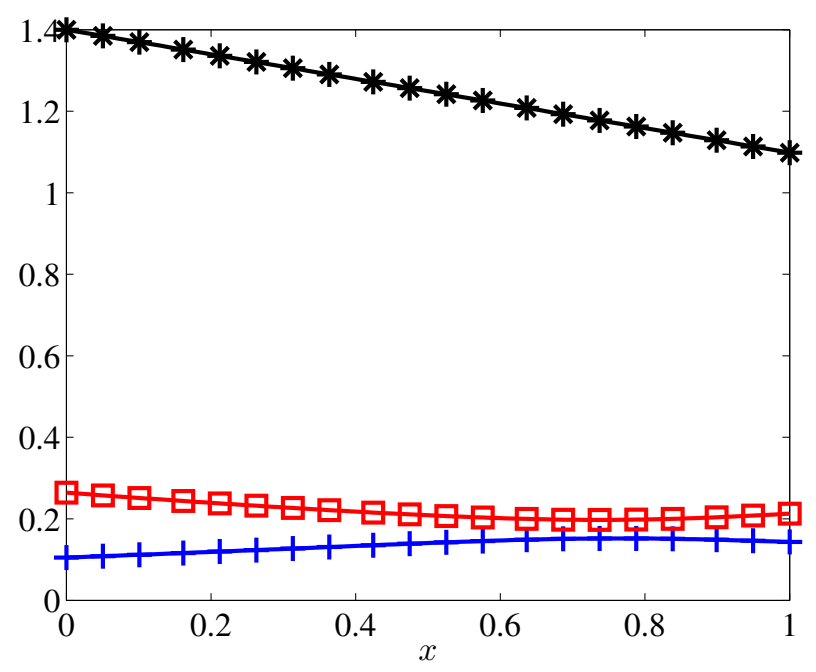

Figure 1: Evolution of the eigenvalues of (94) as a function of $x$ (square and cross markers), and of the determinant of $P(x)$ in 103 (star marker) for Example 2

The verification of the positive definiteness of matrix (94) is more delicate due to its dependence on $x$. Figure 1 shows the evolution of the eigenvalues of $M_{2}(x)$ and the determinant of matrix 103 , which remain positive for all $x \in[0,1]$.

\subsection{Compensation in the Output of In-Domain and Boundary Disturbances}

Let us assume that there exist some disturbances $d_{1}, d_{2} \in C^{1}([0,1])$ on the right-hand side of (11), (12), respectively and some disturbances $d_{3}, d_{4} \in \mathbb{R}$ on the right-hand side of [15), (16), respectively. The error system (87)-(90) becomes

$$
\begin{aligned}
\tilde{u}_{t}+\varepsilon_{1}(x) \tilde{u}_{x} & =\gamma_{1}(x) \tilde{v}+d_{1}(x) \\
\tilde{v}_{t}-\varepsilon_{2}(x) \tilde{v}_{x} & =\gamma_{2}(x) \tilde{u}+d_{2}(x) \\
\tilde{u}(0, t) & =q \tilde{v}(0, t)+d_{3} \\
\tilde{v}(1, t) & =\tilde{U}(t)+d_{4},
\end{aligned}
$$

with $\tilde{U}(t)$ and $\tilde{\eta}(t)$ given by (91) and (92) respectively. The equilibrium of the perturbed system (142)-(144) and (90) is the solution of the following ordinary differential equation

$$
Z^{\prime}(x)=F(x) Z(x)+G(x)
$$

where $F(x)=\left[\begin{array}{cc}0 & \frac{\gamma_{1}(x)}{\varepsilon_{1}(x)} \\ -\frac{\gamma_{2}(x)}{\varepsilon_{2}(x)} & 0\end{array}\right]$ and $G(x)=\left[\begin{array}{c}\frac{d_{1}(x)}{\varepsilon_{1}(x)} \\ -\frac{d_{2}(x)}{\varepsilon_{2}(x)}\end{array}\right]$, with boundary conditions

$$
\begin{aligned}
& Z_{1}(0)=d_{3} \\
& Z_{2}(0)=0 .
\end{aligned}
$$


The ordinary differential equation (146) together with the boundary conditions $(147),(148)$ is a well-posed initial value problem for $x$. The equilibrium depends on $d_{1}, d_{2}$ and $d_{3}$. Let us denote this equilibrium by $\tilde{u}_{s s}\left(x ; d_{1}, d_{2}, d_{3}\right), \tilde{v}_{s s}\left(x ; d_{1}, d_{2}, d_{3}\right)$. From 145$)$ it follows that the equilibrium value of $\tilde{U}$, namely $\tilde{U}_{s S}$, satisfies

$$
\tilde{U}_{s s}=\tilde{v}_{s s}\left(1 ; d_{1}, d_{2}, d_{3}\right)-d_{4} .
$$

Using $(91)$, and 148 with $Z=\left[\tilde{u}_{s s}, \tilde{v}_{s s}\right]^{\top}$, it follows from 149 that the equilibrium value of $\tilde{\eta}$, namely $\tilde{\eta}_{s s}$, satisfies

$$
\tilde{\eta}_{s s}=-\frac{\tilde{v}_{s s}\left(1 ; d_{1}, d_{2}, d_{3}\right)-d_{4}}{k_{I}}
$$

Let us define

$$
\begin{aligned}
\bar{u}(x, t) & =\tilde{u}(x, t)-\tilde{u}_{s s}\left(x ; d_{1}, d_{2}, d_{3}\right) \\
\bar{v}(x, t) & =\tilde{v}(x, t)-\tilde{v}_{s s}\left(x ; d_{1}, d_{2}, d_{3}\right) \\
\bar{\eta}(t) & =\tilde{\eta}(t)-\tilde{\eta}_{s s} .
\end{aligned}
$$

Using $\left[146\right.$ with $Z=\left[\tilde{u}_{s s}, \tilde{v}_{s s}\right]^{\top}$ together with $[142,, 143$ it is shown that the variables $\bar{u}$ and $\bar{v}$ satisfy

$$
\begin{aligned}
\bar{u}_{t}+\varepsilon_{1}(x) \bar{u}_{x} & =\gamma_{1}(x) \bar{v} \\
\bar{v}_{t}-\varepsilon_{2}(x) \bar{v}_{x} & =\gamma_{2}(x) \bar{u} .
\end{aligned}
$$

Setting $x=0$ in (151), (152), and using (144), (147), and (148) we get that

$$
\bar{u}(0, t)=q \bar{v}(0, t) .
$$

Setting $x=1$ in 152 ) and using (145), (91), and (150) we get $\bar{v}(1, t)=-k_{P} \tilde{v}(0, t)-k_{I} \tilde{\eta}(t)+k_{I} \tilde{\eta}_{s s}$. Using (152) for $x=0$ together with $(148)$ and $(153)$ we arrive at

$$
\bar{v}(1, t)=-k_{P} \bar{v}(0, t)-k_{I} \bar{\eta}(t) .
$$

Using (153) and the fact that

$$
\bar{v}(0, t)=\tilde{v}(0, t),
$$

relation 92 becomes

$$
\dot{\bar{\eta}}(t)=\bar{v}(0, t) .
$$

Under the assumptions of Theorem 2 the zero equilibrium of $(154)-(157)$ and $(159)$ is exponentially stable. 


\section{Simulations}

\subsection{Trajectory Generation for a Wave PDE}

In this subsection, we illustrate our trajectory generation methodology with a wave PDE of the form (70)-(72). We choose the parameters of the system as

$$
\begin{aligned}
\varepsilon & =1 \\
h & =1 \\
b & =-1 \\
g & =0 .
\end{aligned}
$$

The reference for the output is chosen as $\zeta(t)=\sin (3 t)$. As in Example 1 , this choice of parameters gives $c_{2}=c_{4}=0$, and hence $\gamma_{1}=0$. Therefore, using relations (65)-(68) we obtain

$$
\begin{aligned}
L^{\alpha \alpha}(x, \xi) & =0 \\
L^{\alpha \beta}(x, \xi) & =0 \\
L^{\beta \alpha}(x, \xi) & =-\frac{1}{2} \exp \left(\frac{x+\xi}{2}\right) \\
L^{\beta \beta}(x, \xi) & =-\frac{1}{2} \exp \left(\frac{x-\xi}{2}\right) .
\end{aligned}
$$

The reference trajectory $z^{r}$ for system (70)-(72) is given by

$$
\begin{aligned}
z^{r}(x, t)= & \frac{1}{37}(19 \sin (3 t+3 x)-3 \exp (x) \cos (3 t-3 x)+3 \cos (3 t+3 x) \\
& +18 \exp (x) \sin (3 t-3 x))
\end{aligned}
$$

which gives the following reference input

$$
\begin{aligned}
W^{r}(t)= & \frac{57}{37}(\cos (3 t+3)-\exp (1) \cos (3 t-3)) \\
& +\frac{9}{37}(\exp (1) \sin (3 t-3)-\sin (3 t+3)) .
\end{aligned}
$$

Figure 2 shows the evolution of the reference trajectory $z^{r}$. Figure 3 shows the evolution of the spatial derivative of $z^{r}$ and, in particular, the control effort $W^{r}(t)=z_{x}^{r}(1, t)$ given by (169).

\subsection{Trajectory Tracking}

In this subsection, a simulation study for the system from Examples 1 and 2 is presented. The numerical approximation of the solution is computed with a two-step variant of the Lax-Friedrichs $(\mathrm{LxF})$ method [30]. The reference for the output is chosen as $y^{r}(t)=\cos (t)$. We add disturbances at the right-hand side of (61), 62) given by

$$
\begin{aligned}
& d_{1}(x)=0.5 \exp (x) \\
& d_{2}(x)=\cos (2 x)
\end{aligned}
$$




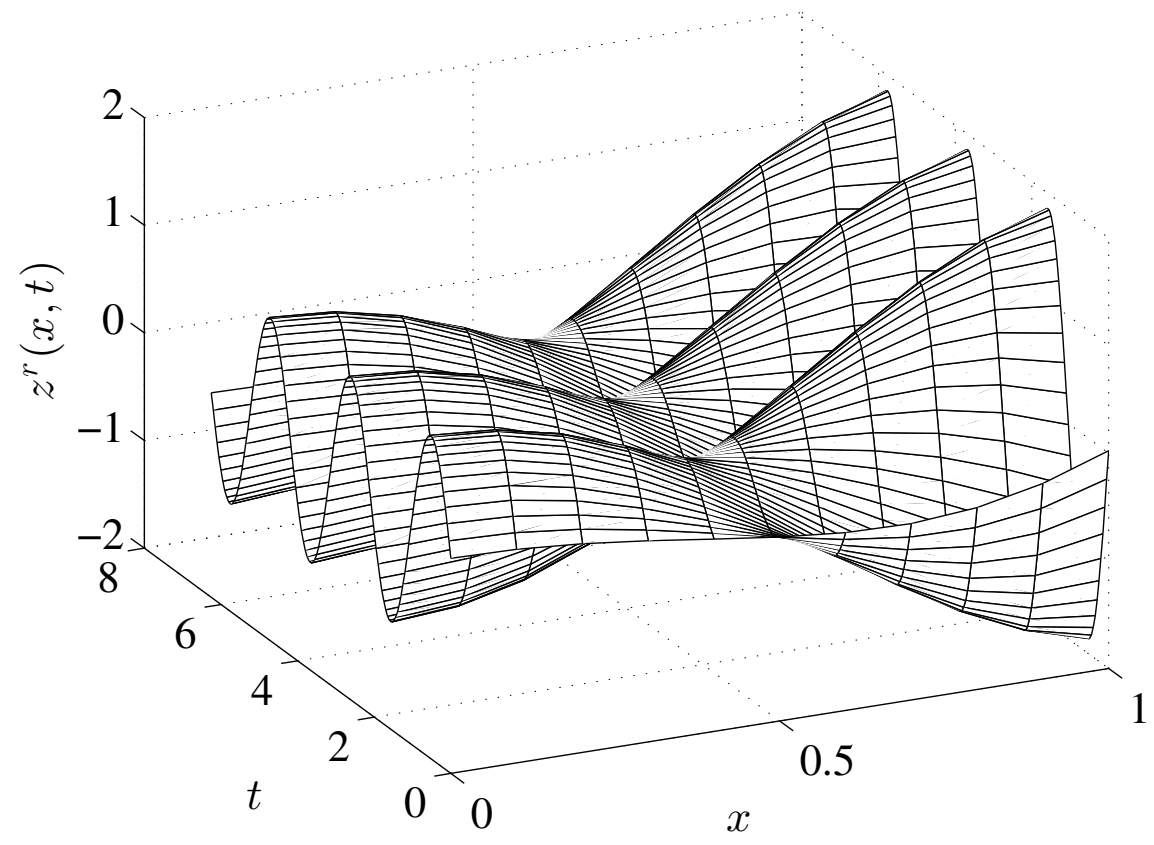

Figure 2: Solution to the trajectory generation problem for system $(70-(72)$ with parameters $[160]-163)$.

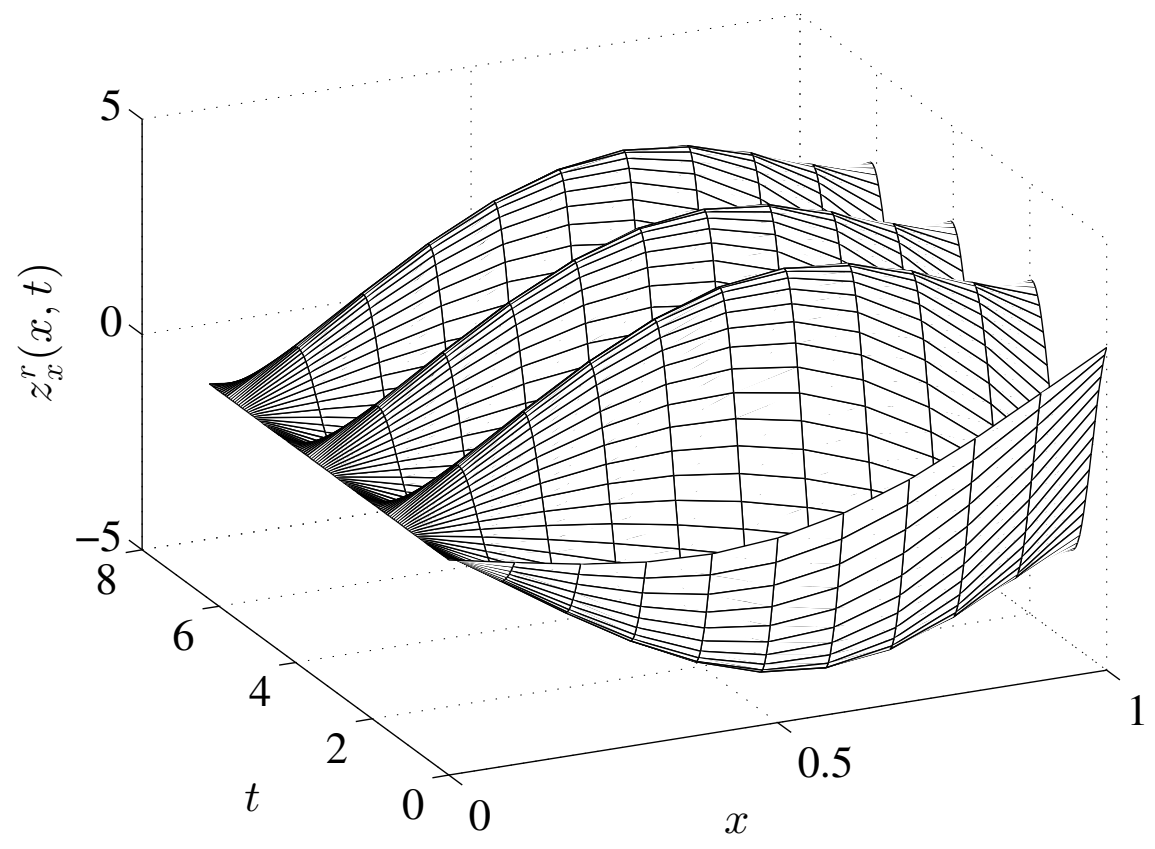

Figure 3: The spatial derivative of the reference trajectory of Figure 2. Note in particular the reference input $W^{r}(t)=$ $z_{x}^{r}(1, t)$ given by 169 . 
together with constant additive disturbances on the boundary conditions (63), 64) given by

$$
\begin{aligned}
& d_{3}=0.5 \\
& d_{4}=0.5 .
\end{aligned}
$$

The initial conditions for $u$ and $v$ are chosen as the reference initial conditions given by (19), (20) for $t=0$, perturbed by spatially-varying errors as

$$
\begin{aligned}
& u(x, 0)=u^{r}(x, 0)+\sin (x) \\
& v(x, 0)=v^{r}(x, 0)+\cos (x),
\end{aligned}
$$

and the initial condition for $\tilde{\eta}$ is chosen such that $U(0)=v(1,0)$, that is,

$$
\tilde{\eta}(0)=\frac{U^{r}(0)-v(1,0)+k_{P}\left(v^{r}(0,0)-v(0,0)\right)}{k_{I}} .
$$

Figure 4 shows that the output of the system $v(0, t)$ follows the desired trajectory under the PI controller given by

$$
\begin{aligned}
U(t)= & \cos \left(t+\frac{1}{6}\right)+\frac{3}{229}\left(\exp \left(-\frac{1}{45}\right) \sin (t)-\exp \left(-\frac{1}{15}\right) \sin \left(t-\frac{1}{3}\right)\right) \\
& +\frac{2}{1145}\left(\exp \left(-\frac{1}{45}\right) \cos (t)-\exp \left(-\frac{1}{15}\right) \cos \left(t-\frac{1}{3}\right)\right) \\
& +\frac{6}{241}\left(\exp \left(\frac{1}{45}\right) \sin \left(t+\frac{1}{6}\right)-\exp \left(-\frac{1}{45}\right) \sin (t)\right) \\
& +\frac{8}{1205}\left(\exp \left(\frac{1}{45}\right) \cos \left(t+\frac{1}{6}\right)-\exp \left(-\frac{1}{45}\right) \cos (t)\right) \\
& -k_{P}(v(0, t)-\cos (t))-k_{I} \tilde{\eta}(t),
\end{aligned}
$$

with gains 138), 139), and $\dot{\tilde{\eta}}(t)=v(0, t)-\cos (t)$. One can also observe that with only a $\mathrm{P}$ controller (i.e., when $k_{I}=0$ in (177)) there is a steady-state tracking error. Figure 5 shows the evolution of the state $v$.

\section{Conclusions}

We presented solutions to the trajectory generation and tracking problems for general $2 \times 2$ systems of first-order linear hyperbolic PDEs. We solved the motion planning problem with backstepping and the trajectory tracking problem with PI control. We proved exponential stability of the closed-loop system by constructing a Lyapunov functional.

\section{Acknowledgments}

The authors are deeply grateful to Antoine Girard and Christophe Prieur for many constructive suggestions. 


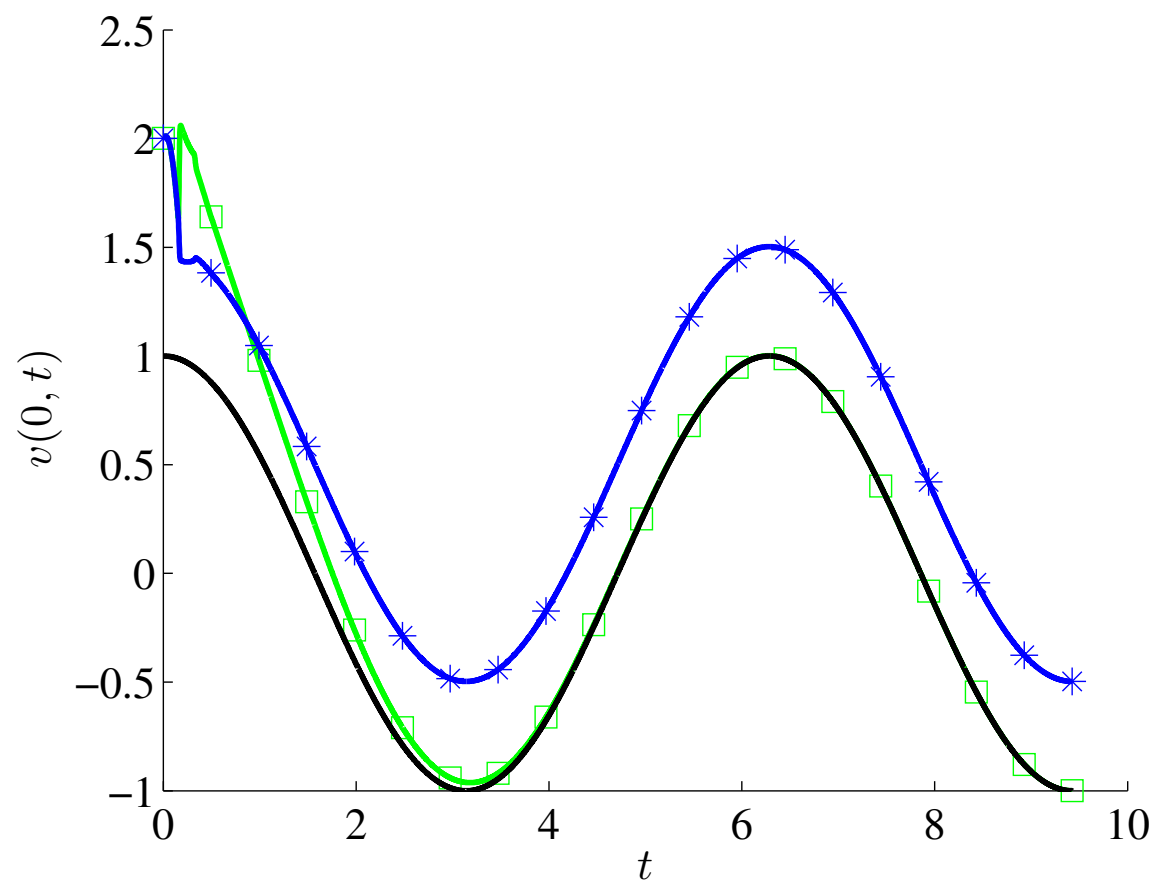

Figure 4: The output $v(0, t)$ of system 61 - 64) with parameters $134-(137)$ under the control law 177 with gains (138), 1139] (square marker) and with gains (138), $k_{I}=0$ (star marker) for the initial conditions (174)-(176). The single line is the reference output $y^{r}(t)=\cos (t)$.

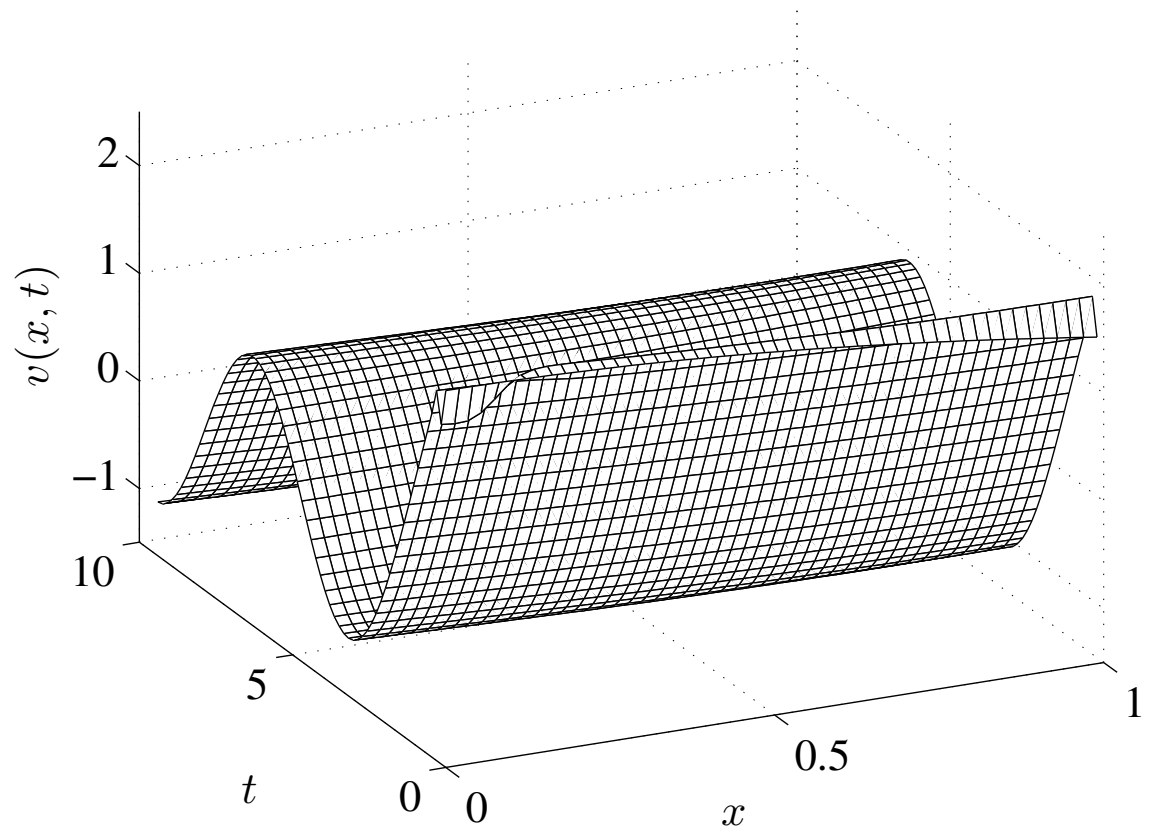

Figure 5: Evolution of the state $v$ of system (61)- 64 with parameters 134 - 137) under the control law (177) with gains (138), 139) for the initial conditions (174)-1176). 
[1] O. M. Aamo, Disturbance rejection in $2 \times 2$ linear hyperbolic systems, IEEE Transactions on Automatic Control, 58 (2013) 1095-1106.

[2] G. Bastin and J.-M. Coron, On boundary feedback stabilization of non-uniform linear $2 \times 2$ hyperbolic systems over a bounded interval, Systems \& Control Letters, 60 (2011) 900-906.

[3] N. Bekiaris-Liberis and M. Krstic, Compensation of wave actuator dynamics for nonlinear systems, IEEE Transactions on Automatic Control, 59 (2014) 1555-1570.

[4] J. Cochran and M. Krstic, Motion planning and trajectory tracking for the 3-D Poiseuille flow, Journal of Fluid Mechanics, 626 (2009) 307-332.

[5] J.-M. Coron, B. d'Andréa-Novel, and G. Bastin, A strict Lyapunov function for boundary control of hyperbolic systems of conservation laws, IEEE Transactions on Automatic Control, 52 (2007) 2-11.

[6] J.-M. Coron, G. Bastin, and B. d'Andréa-Novel, Dissipative boundary conditions for one dimensional nonlinear hyperbolic systems, SIAM Journal on Control and Optimization, 47 (2008) 1460-1498.

[7] J.-M. Coron, R. Vazquez, M. Krstic, and G. Bastin, Local exponential $H^{2}$ stabilization of a $2 \times 2$ quasilinear hyperbolic system using backstepping, SIAM Journal on Control and Optimization, 51 (2013) 2005-2035.

[8] C. Curro, D. Fusco, and N. Manganaro, A reduction procedure for generalized Riemann problems with application to nonlinear transmission lines, Journal of Physics A: Mathematical and Theoretical, 44 (2011) 335205.

[9] J. de Halleux, C. Prieur, J.-M. Coron, B. d'Andréa-Novel, and G. Bastin, Boundary feedback control in networks of open channels, Automatica, 39 (2003) 1365-1376.

[10] A. Diagne, G. Bastin, and J.-M. Coron, Lyapunov exponential stability of linear 1-D hyperbolic systems of balance laws, Automatica, 48 (2012) 109-114.

[11] F. Di Meglio, M. Krstic, R. Vazquez, and N. Petit, Backstepping stabilization of an underactuated $3 \times 3$ linear hyperbolic system of fluid flow transport equations, American Control Conference, 2012.

[12] F. Di Meglio, R. Vazquez, and M. Krstic, Stabilization of a system of $n+1$ coupled firstorder hyperbolic linear PDEs with a single boundary input, IEEE Transactions on Automatic Control, 58 (2013) 3097-3111.

[13] V. Dos Santos, G. Bastin, J.-M. Coron, and B. d'Andréa-Novel, Boundary control with integral action for hyperbolic systems of conservation laws: Stability and experiments, Automatica, 44 (2008) 1310-1318.

[14] V. Dos Santos and C. Prieur, Boundary control of open channels with numerical and experimental validations, IEEE Transactions on Control Systems Technology, 16 (2008) 1252-1264. 
[15] F. Dubois, N. Petit, and P. Rouchon, Motion planning and nonlinear simulations for a tank containing a fluid, European Control Conference, Karlsruhe, Germany, 1999.

[16] S. Fan, M. Herty, and B. Seibold, Comparative model accuracy of a data-fitted generalized Aw-Rascle-Zhang model, Networks \& Heterogeneous Media, 9 (2014) 239-268.

[17] M. Fliess, H. Mounier, P. Rouchon, and J. Rudolph, A distributed parameter approach to the control of a tubular reactor: A multi-variable case, IEEE Conference on Decision and Control, Tampa, FL, 1998.

[18] P. Goatin, The Aw-Rascle vehicular traffic flow model with phase transitions, Math. Comp. Modeling, 44 (2006) 287-303.

[19] M. Gugat, M. Dick, and G. Leugering, Gas flow in fan-shaped networks: Classical solutions and feedback stabilization, SIAM Journal on Control and Optimization, 49 (2011) 2101-2117.

[20] M. Gugat and M. Herty, Existence of classical solutions and feedback stabilization for the flow in gas networks, ESAIM: Control, Optimisation and Calculus of Variations, 17 (2011) $28-51$.

[21] H. Hochstadt, Integral Equations. Wiley-Interscience, New-York, 1973.

[22] L. Hu, F. Di Meglio, R. Vazquez, and M. Krstic, Control of homodirectional and general heterodirectional linear coupled hyperbolic PDEs, under review. Preprint submitted to arxiv on April 2015. Available at: arxiv.org/pdf/1504.07491v1.pdf.

[23] M. Krstic and A. Smyshlyaev, Boundary Control of PDEs. Adv. Des. Control 16, SIAM, Philadelphia, 2008.

[24] T.-T. Li, Classical solutions for quasilinear hyperbolic systems, John Wiley \& Sons. Chichester, 1994.

[25] X. Litrico and V. Fromion, Boundary control of hyperbolic conservation laws using a frequency domain approach, Automatica, 45 (2009) 647-656.

[26] N. Petit and P. Rouchon, Dynamics and solutions to some control problems for water-tank systems, IEEE Transactions on Automatic Control, 47 (2002) 594-609.

[27] N. Petit and P. Rouchon, Flatness of heavy chain systems, SIAM Journal on Control and Optimization, 40 (2001) 475-495.

[28] C. Prieur, Control of systems of conservation laws with boundary errors, Networks \& Heterogeneous Media, 4 (2009) 393-407.

[29] C. Prieur, J. Winkin, and G. Bastin, Robust boundary control of systems of conservation laws, Mathematics of Control, Signals, and Systems, 20 (2008) 173-197.

[30] L. F. Shampine, Solving Hyperbolic PDEs in MATLAB, Applied Numerical Anal. \& Comput. Math., 2 (2005) 346-358. 
[31] A. Siranosian, M. Krstic, A. Smyshlyaev, and M. Bement, Motion planning and tracking for tip displacement and deflection angle for flexible beams, Journal of Dynamic Systems, Measurement, and Control, 131 (2009) 031009.

[32] B. S. Tang and M. Krstic, Sliding mode control to stabilization of linear $2 \times 2$ hyperbolic systems with boundary input disturbance, American Control Conference, 2014.

[33] R. Vazquez and M. Krstic, Marcum Q-functions and explicit kernels for stabilization of linear hyperbolic systems with constant coefficients, Systems \& Control Letters, 68 (2014) 33-42.

[34] R. Vazquez, M. Krstic, and J.-M. Coron, Backstepping boundary stabilization and state estimation of a $2 \times 2$ linear hyperbolic system, IEEE Conference on Decision and Control, Orlando, FL, 2011.

[35] R. Vazquez, M. Krstic, J.-M. Coron, and G. Bastin, Collocated output-feedback stabilization of a $2 \times 2$ quasilinear hyperbolic system using backstepping, American Control Conference, 2012. 